\title{
Oteiza en movimiento: una herramienta tecnológica para el aprendizaje participativo en el Museo Oteiza
}

Oteiza in motion a tool for a collaborative learning at the Oteiza museum

\author{
> Alfredo Pina \\ Univ. Pública de Navarra \\ pina@unavarra.es \\ > Benoit Bossavit \\ Univ. Pública de Navarra \\ bossavit@unavarra.es
}

\author{
> Isabel Sanchez Gil \\ Univ. Pública de Navarra \\ isabel.sanchezgil@unavarra.es \\ > Aitziber Urtasun \\ Museo Oteiza \\ didactica@museooteiza.org
}

\begin{abstract}
This paper presents an interdisciplinary work done at the Museum Oteiza near Pamplona, Spain during 2012 and 2013 . The main goal is the use of low cost devices that allows natural interaction, Virtual and Augmented Realities to get the general public closer to the works and concepts of Oteiza through interactive serious games. The most difficult Oteiza's concepts to understand are those related with the empty space or with time \& space. We propose three different activities, shaped as serious game, that use the whole body to interact and play with these concepts. The result is a facility that has been installed at the entrance of the Museum and that can be used for free, before or after a visit to the museum. These activities were presented to 4 experts in Oteiza and the results are discussed. In addition, we gathered more comments after several groups of children tried the application in the museum.
\end{abstract}

Keywords: Realidad Virtual, Interacción natural, Museos, Aprendizaje informal

\section{Introducción}

El objetivo general que se había planteado en este proyecto ("Oteiza para tod@s”) era generar una nueva línea de trabajo (en arte, educación y tecnología) y establecer una plataforma de trabajo con el fin de difundir la obra de Oteiza en diferentes ámbitos (itinerarios educativos, formales e informales, en el museo y fuera de él) y para diferente público (adultos, niños, escolares, con necesidades especiales).

El equipo de trabajo interdisplinar que ha participado en este proyecto, Arte y Educación, Tecnología, Tecnología y Educación, ha desarrollado un producto, denominado "Oteiza en Movimiento", que da respuesta a los objetivos planteados en este proyecto de investigación de la fundación Museo Oteiza (http://www.museooteiza.org/). El Museo Oteiza alberga la colección personal del reconocido escultor y artista integral (Jorge Oteiza, Orio, Gipuzkoa, España 1908 - Donostia, Gipuzkoa, España 2003), compuesta por 1.650 esculturas, 2.000 piezas de su laboratorio experimental, además de una extensa presencia de dibujos y collages. Este proyecto ha sido financiado por Kutxa obra social (Caja de Ahorros y Monte de Piedad de Gipuzkoa y San Sebastián, en euskera Gipuzkoa eta Donostiako Aurrezki Kutxa, o, simplemente Kutxa, como es más conocida, es una caja de ahorros guipuzcoana. Kutxa ('caja' en euskera) es la marca comercial que utiliza.

Todo el desarrollo del mismo se ha hecho en estrecha colaboración con la sección de didáctica del Museo Oteiza.

\section{Jorge Oteiza}

Jorge Oteiza representa uno de los artistas más importantes del panorama contemporáneo español y un líder indiscutible de la renovación estética del arte del siglo XX (Pelay, 1978) (Alvarez et al, 2003). Su infancia se desarrolla en el País Vasco, y tras completar sus estudios de bachillerato sus pasos se encaminan hacia Madrid donde comienza sus estudios de Medicina y toma verdadero contacto con el arte de vanguardia.

En 1935 el escultor vasco decide abordar el que sería el viaje más importante de su vida. Se traslada a Buenos Aires desde donde comienza un enriquecedor periplo que le llevará a Bolivia, Argentina, Chile Colombia y Uruguay. Gracias a la relación del artista con la escultura megalítica americana (Muñoz, 2006) se definirá en las líneas teóricas de su trabajo. Oteiza otorga al arte una función práctica en la sociedad y la obra de arte, en sí misma, debe poseer un contenido espiritual. El artista, desde la herramienta del arte, puede proporcionar al mundo una metafísica para la conducta y una solución como refugio, como servicio espiritual.

Su vuelta a España, en 1948, le lleva a desarrollar una de las carreras artísticas más completas del panorama español (algunos de sus éxitos le devolverán a América, como su triunfo en la Bienal de Sao Paulo de 1957), convirtiéndose en un referente de la vanguardia artística del siglo XX. En 1959 abandona públicamente la escultura para desarrollar otros lenguajes como la poesía, el cine o la arquitectura, y comienza así una nueva etapa en la que centra sus esfuerzos en la elaboración de nuevas escuelas y propuestas pedagógicas en el País Vasco.

Los últimos años de su vida le acercan a un pequeño pueblo de Navarra, Alzuza, donde se retira a meditar y escribir, y donde actualmente se encuentra la Fundación Museo Jorge Oteiza. La obra 
escultórica de Oteiza transita entre las primeras vanguardias del arte moderno (cubismo, constructivismo o suprematismo, entre otras) integrando en el desarrollo formal de la obra conceptos propios de las matemáticas, la física o la filosofía. Es por ello, que su estudio con escolares resulta complejo y herramientas de acercamiento como "Oteiza en movimiento” resultan de enorme importancia (Urtasun, 2006).

\section{Trabajo previo}

El uso de la Microsoft Kinect como dispositivo para implementar interacción natural es cada vez más común. En el ámbito de los museos tenemos un ejemplo de utilización en una instalación que versa sobre arqueología Etrusca en los Museos Vaticanos (Hupperetz et al, 2012). En este caso este dispositivo y diferentes acciones de los brazos permiten navegar a través de una Realidad Virtual que representa diferentes capas de información arqueológica. Asimismo el uso de otros dispositivos móviles son cada vez más utilizados en los Museos para facilitar la comprensión de las obras y aumentar las posibilidades de aprendizaje y participación durante las visitas (Chia-Chen Chen and Tien-Chi Huang, 2012). Otro aspecto relevante hoy en día respecto a los museos, es que más allá de las visitas individuales o colectivas, se organizan visitas escolares. En general suelen ser visitas desde las escuelas hacia los museos. Sin embargo este tipo de tecnologías ubicuas y transportables facilitan que esta participación pueda ser en ambos sentidos, y que estas instalaciones puedan llevarse a las escuelas (Jocelyn Wishart and Pat Triggs, 2010).

En el trabajo que presentamos intentamos aunar todas estas posibilidades tecnológicas con aprendizajes escolares tanto en la escuela como fuera de ella, en este caso en un Museo. El resto del artículo explica la metodología de trabajo y los materiales utilizados. Describe los resultados alcanzados así como la evaluación con expertos llevada a cabo y la experimentación con público en el Museo. La última sección concluye y plantea líneas de futuro.

\section{Metodología y materiales}

Como se ha expuesto anteriormente este proyecto proponía reflexionar sobre la obra de Oteiza desde tres puntos de vista diferentes pero que creíamos complementarios, Arte, Educación y Tecnología.

Por un lado algunos de los planteamientos artísticos de Oteiza son difíciles de transmitir al público en general y a los niñ@s en particular, véase aquellos conceptos relacionados con la desocupación espacial, o bien aquellos que tienen que ver con espacio y tiempo. Por otro lado, desde un punto de vista educativo y pensando en actividades educativas validas tanto en contextos formales como informales nuestra "transposición didáctica" ha ido encaminada a generar lo que hoy se entiende como "juego serio", y por lo tanto hemos creado una aplicación informática que permite al usuario jugar con algunos de los planteamientos artísticos de Oteiza.

Finalmente el tercer aspecto importante que queríamos lograr es que la herramienta desarrollada fuera de un coste razonable, que se pudiera utilizar en diferentes espacios (en el museo o fuera de él) y que su utilización fuera accesible e inclusiva.

Por lo explicado anteriormente, el producto a desarrollar se puede definir como una aproximación a la obra y procesos de Jorge Oteiza a partir de la manipulación "libre y participativa" de algunas piezas representativas del artista utilizando visualización 2D/3D, Realidad Aumentada, Realidad Virtual e interacción natural. El equipo de trabajo ha tenido en cuenta en este diseño las características de implantación del mismo: accesibilidad, "lowcost", implantación fija en el museo, posibilidad de "trasportar" el producto para su uso en Escuelas o similares fuera del museo.

Hemos elegido para ello una tecnología basada en lo que se entiende como "interacción natural", que permite que el usuario interaccione con la aplicación mediante su cuerpo. La implementación se ha llevado a cabo con un dispositivo de videojuego como es la Kinect, y la aplicación admite su uso en 2D o en 3D (utilizando TV 3D convencional).

El juego tiene tres apartados bien definidos; dos de ellos permiten descubrir y explorar los "espacios desocupados" de las obras de Oteiza y el otro permite experimentar con la tercera y cuarta dimensión, i.e. con tiempo y espacio. La interacción del usuario es mediante el cuerpo y facilita la selección y manipulación de objetos en un espacio 3D.

La metodología que se ha seguido para el desarrollo ha consistido básicamente en reflexionar sobre aquellas partes de la obra artística de Oteiza que eran difíciles de transmitir (espacio, 3D y tiempo) y en analizar las posibilidades que nos daba la tecnología (sobre todo para interaccionar y modelar objetos y entornos); a partir de estas reflexiones hemos ido formalizando los itinerarios educativos. Cada uno de los juegos desarrollados se ha ido validando con la responsable de la sección de didáctica del Museo Oteiza, Aitziber Urtasun, de manera que la herramienta obtenida pueda ser utilizada en las actividades didácticas propias del museo.

\section{Resultados}

Como resultado del proyecto se ha hecho una instalación de la herramienta desarrollada en el Museo que es completamente funcional. Tanto la herramienta como la instalación han sido validadas técnica, educativa y artísticamente por el equipo de trabajo y por la sección de didáctica del museo. Se han hecho ya algunas experimentaciones fuera del museo, en un centro escolar de Pamplona.

Desde el punto de vista de visualización, queremos asegurar el máximo de posibilidades de trabajo, tanto en $2 \mathrm{D}$ como en $3 \mathrm{D}$, y con la mayor variedad posible de dispositivos asequibles para el museo y/o para las escuelas:

Podemos trabajar en 2D:

- Proyector/Monitor

- TV

Podemos trabajar en 3D:

- Proyector/Monitor anáglifo (con gafas cromáticas)

- TV 3D con gafas pasivas (polarizadas)

Desde el punto de vista de interacción queremos poder asegurar que el máximo posible de usuari@s puedan manipular, seleccionar piezas y navegar alrededor de las piezas.

Para la interacción utilizamos un sensor Microsoft Kinect, tal y como se ha comentado anteriormente, que nos permite trabajar con diferentes gestos, posturas, mirada, etc....

Además hemos probado a utilizar marcadores para poder combinarlos con los gestos y posturas, de manera que enriquecemos y flexibilizamos las posibilidades de interacción por parte del usuario. 


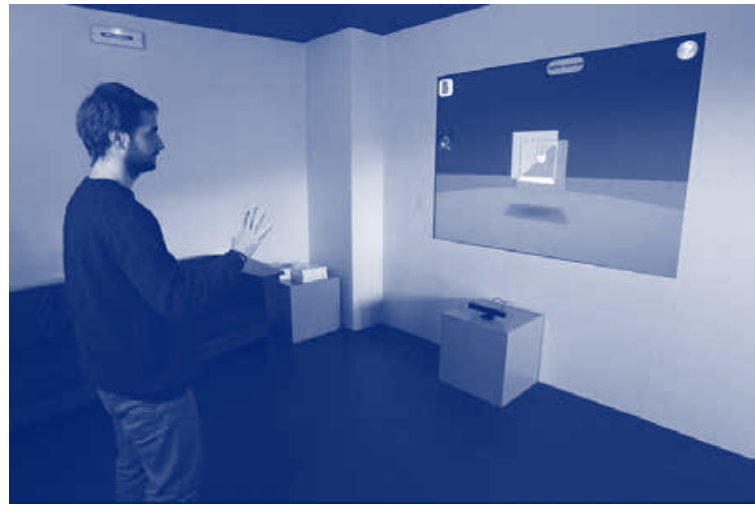

Figura 1: Instalación en el Museo Oteiza.

Sin embargo y debido a la poca precisión del sensor, el uso de los marcadores es confuso y de momento no hemos podido validar y generalizar su uso.

\section{Evaluación con expertos}

La instalación se puso en marcha en diciembre de 2013 de manera experimental. A lo largo de este año 2014 ha estado disponible en el Museo. Estamos a la espera de hacer una evaluación en relación al uso con el público en general y con los grupos de escolares. Sin embargo ya hemos tenido una primera evaluación con expertos que nos está permitiendo hacer la segunda versión del software. Esta evaluación se hizo en febrero de 2014 y han participado 4 expertos, uno por parte del Museo, dos de la universidad de Barcelona y un cuarto de la Universidad Pública de Navarra. Participaron además dos de los desarrolladores del proyecto. La evaluación de desarrolló de la siguiente manera. Durante $1 \mathrm{~h}$ aproximadamente se hizo una visita al Museo. A continuación se les explicó de manera muy breve el funcionamiento de la aplicación y los cuatro expertos estuvieron interactuando con la misma durante $1 \mathrm{~h}$ aproximadamente (unos 15 minutos por persona). A partir de ahí se estableció un intercambio de ideas que supuso una valoración cualitativa de la aplicación por parte de los expertos. A continuación describimos sus principales aportaciones, organizadas en comentarios generales, aspectos de usabilidad y análisis por actividades.

\section{Comentarios generales}

Todos están de acuerdo en que a priori con el programa tal y como esta, se trabaja el razonamiento abstracto, que el juego "conduce" al usuario a un logro previsto y que el juego se podría plantear como un aprendizaje en este ámbito.

En relación a la navegación en el juego los expertos (todos) piensan que el hecho de tener niveles (de dificultad) que hay que ir superando para avanzar en el juego puede no ser siempre la mejor estrategia. Por ejemplo plantean que en vez de superar niveles, se pueda ir generando, de alguna manera, comprensión de los conceptos de Oteiza, de sus procesos de trabajo y de la relación materia espacio, a medida que el usuario interacciona con la herramienta.

Todos coinciden en que sería interesante que el usuario pudiera construir algo, que no sea totalmente prefijado por el programa
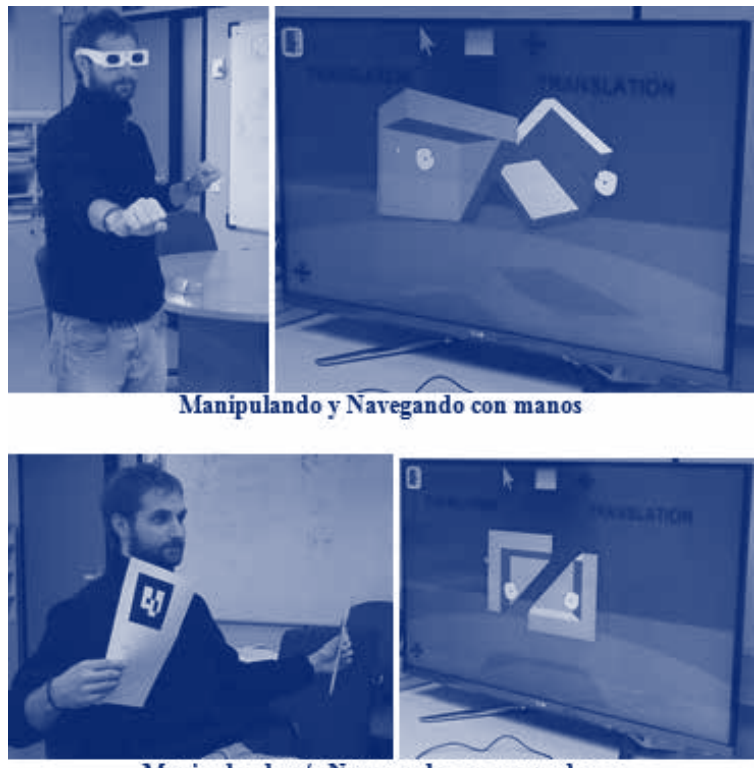

Manipulando y/o Navegando con marcadores

Figura 2: Interacción natural con manos y/o con marcadores. Se puede ver un video demostrativo de la herramienta en: http://youtu.be/dDqQrGc173o

(como ocurre ahora...). Como alternativa a esta observación, todos plantean que pueda haber (por ejemplo para cada actividad) la posibilidad de seguir el juego por un lado, y por otro la posibilidad de crear por parte del usuario su propio modulo. Esto se ve claramente en el caso de la esfera tal y como se indica más adelante.

Como principal conclusión se plantea que es importante tener claro cuál es el objetivo y uso de la herramienta.....y las preguntas surgen... ¿ ¿Solo es un complemento a la visita?....¿tiene sentido para una visita sin guía?....¿qué limitaciones tiene?

\section{Usabilidad}

La interacción en el espacio 3D mediante movimientos de brazos y/o manos es razonablemente intuitiva pero difícil de explicar. Por ello el manual o tutorial que lo explica no convence a los expertos, ya que a la vez habla del juego y de la manipulación. Estos plantean separar y crear un tutorial específico de movimientos por un lado y por otro lado el tutorial de juegos.

Estos tutoriales están hechos con textos e iconos, pero los expertos consideran que se pueden mejorar. Todos proponen añadir animaciones y/o videos para explicar cómo se hacen las translaciones y rotaciones, así como ser rigurosos y precisos explicando las rotaciones y translaciones.

Otro aspecto resaltado por los expertos es que no siempre hay coherencia en el tutorial (y en la aplicación): por ejemplo la posición de los iconos de ayuda se pueden confundir con iconos para pasar al modo de análisis en el apartado de construcción (una de las actividades), ya que en ese caso parece el "target" y no el paso a "modo de análisis".

La aplicación no presenta sonido y uno de los expertos pregunta porque. No parece algo muy relevante, pero un sonido acorde podría ser interesante. En este momento de la discusión surgen otras ideas como la de ańadir más fotografías de obras de Oteiza o ańadir frases célebres y/o significativas del propio autor en la aplicación. 
Finalmente un experto pregunta porque las puntuaciones son numéricas y no gráficas. La idea detrás de esta pregunta es que quizás sea interesante reforzar conceptos también con la puntuación del juego.

\section{Actividad de desocupación del espacio}

En esta actividad se discute sobre el 2D y 3D. Las actividades propuestas están pensadas en 3D y se trabaja en 3D; sin embargo algunas de las soluciones aparecen en 2D (en parte por falta de espacio físico en la pantalla de la aplicación). Todos apuntan que trabajar constantemente en 3D es algo muy importante en la aplicación y piden que se revise este aspecto en toda la aplicación (no solo en esta actividad). También se discute sobre el aspecto de abstracción entre 2D y 3D, pero prevalece la idea de que es importante conseguir una inmersión del usuario en 3D. Y un último comentario al hilo de esta discusión es que en la soluciones finales deberían aparecer los hiperboloides, formas representativas de la desocupación espacial de Oteiza, tal y como se muestran en los cuadernos/esquemas del autor. Sugieren que estos datos como referencia técnico-artística que tiene que ver con la desocupación y las formas que desocupan se muestren al dar la solución

\section{Actividad de activación de la esfera}

Esta actividad basada en interactuar con una esfera 3D formada por diferentes tiras, lo que permite combinar espacio $3 \mathrm{D}$ y tiempo en la actividad. Un experto no ve apropiado el nombre de "activación" y todos apuntan que la introducción del elemento tiempo no es tan necesaria, ya que para Oteiza el componente de tiempo es más bien metafísico.

Por eso la propuesta para esta actividad es separar el videojuego (creación de la esfera a partir de las tiras....) de otra actividad de construcción, que sería: Construir tu propia esfera jugando con las tiras.

Como alternativa a lo que ya hay sugieren que el programa ofrezca comentarios en relación a lo que se va construyendo: por ejemplo si una tira queda en el aire, se puede decir que desde un punto de vista físico no se sostiene y que por lo tanto no se puede fabricar....para esta parte se quitarían los colores. En este caso se diferencian de esta manera el juego (tiras y creación esfera) del de construcción que será mover las tiras hasta obtener una esfera y en ese proceso el sistema "va comentando" el proceso.

\section{Experimentación con niños en el Museo}

El juego ha estado en funcionamiento en el hall principal del museo desde diciembre de 2013 hasta la actualidad. Sigue en funcionamiento y han experimentado con el alrededor de unos 150 niños con edades entre 5 y 14 años.

A lo largo de estos meses se ha mostrado como una herramienta muy útil en la captación de atención de los niños que visitan el museo y que no tienen propuesta lúdicas que favorezcan su visita al mismo.

"Oteiza en movimiento" se compone de tres recorridos. El primero no supone ninguna dificultad, ni siquiera entre los más pequeños. El segundo requiere de más tiempo pero el concepto acaba siendo bien comprendido. El tercero es el que más dificultad genera no por el concepto a trabajar sino porque a nivel práctico los movimientos de la figura resultan más complejos de controlar.
Se ha constatado que los tutoriales resultan largos ya que los niños y niñas no suelen leerlos. Podría estudiarse, en una segunda fase, la posibilidad de introducir tutoriales orales.

El juego también se ha mostrado a profesores de Primaria en las sesiones de formación del profesorado. Se ha demostrado así que la brecha generacional hace patente el uso/no uso de las tecnologías porque casi todos los adultos que lo han probado se han quejado de su dificultad. Algo que apenas ha ocurrido en el caso de los niños y niñas

\section{Conclusiones y líneas futuras}

La primera etapa de este trabajo consistía en desarrollar la aplicación, validarla con la sección de didáctica del museo e instalarla en el propio museo. A partir de aquí queremos ver lo que puede aportar a los visitantes del museo (niños, adultos, grupos escolares, etc....), como actividad formativa, complementaria y participativa.

Por ello, la línea de futuro trabajo en este proyecto es justamente la utilización de la herramienta con diferentes usuarios en el museo y fuera de él, para obtener un feedback amplio de usuarios reales y poder incorporarlo en próximas versiones de la aplicación.

En un primer análisis hemos realizado una sesión de trabajo con expertos en arte y educación que nos ha permitido reflexionar en grupo sobre la herramienta y sus posibles usos. Estamos incorporando este feedback a la segunda versión de la misma, antes de experimentar con los visitantes del museo y/o con escolares.

\section{Agradecimientos}

Agradecemos a KUTXA OBRA SOCIAL la financiación recibida para llevar a cabo esta investigación.

Agradecemos asimismo al Museo Oteiza por la disposición y ayuda para llevar a cabo el trabajo.

\section{Referencias}

Álvarez Martínez, M.Soledad. Jorge Oteiza, pasión y razón. Fundación Museo Jorge Oteiza Fundazio Museoa. Nerea, SanSebastián, 2003

Chia-Chen Chen and Tien-Chi Huang (2012). Learning in a u-Museum: Developing a context-aware ubiquitous learning environment. Computers \& Education, 59(3), 873-883.

Jocelyn Wishart and Pat Triggs (2010). Museum Scouts: exploring how schools, museums and interactive technologies can work together to support learning. Computers \& Education, 54, 669-678.

Muñoz, María Teresa. Edicion crítica de la estatuaria megalítica americana y Carta a los artistas de América. Fundación Museo Jorge Oteiza Fundazio Museoa. Pamplona, 2006.

Pelay Orozco, M., Su vida, su obra, su pensamiento, su palabra. La Gran Enciclopedia Vasca, Bilbao, 1978.

Urtasun Pineda, Aitziber. Guía para educadores. Fundación Museo Jorge Oteiza Fundazio Museoa. Pamplona, 2006.

Wim Hupperetz, Raffaele Carlani, Daniel Pletinckx, Eva Pietroni (2012). Etruscanning 3D project. The 3D reconstruction of the RegoliniGalassi Tombas a research tool and a new approach in storytelling. Virtual Archaeology Review 3(7), 92-96. 